\title{
CANOPY MODELING OF AQUATIC VEGETATION: CONSTRUCTION OF SUBMERGED VEGETATION INDEX
}

\author{
Zhongqi Ma ${ }^{1}$, Guanhua Zhou ${ }^{1, *}$ \\ ${ }^{1}$ School of Instrumentation Science and Opto-electronics Engineering, Beihang Univerisity, Beijing, China - (mzq11171079, \\ zhouguanhua)@buaa.edu.cn
}

Commission III, WG III/10

KEY WORDS: Remote Sensing, Submerged Vegetation, Vegetation Index, Radiative Transfer Model, Sentinel-2A

\begin{abstract}
:
The unique spectral characteristics of submerged vegetation in wetlands determine that the conventional terrestrial vegetation index cannot be directly employed to species identification and parameter inversion of submerged vegetation. Based on the Aquatic Vegetation Radiative Transfer model (AVRT), this paper attempts to construct an index suitable for submerged vegetation, the model simulated data and a scene of Sentinel-2A image in Taihu Lake, China are utilized for assessing the performance of the newly constructed indices and the existent vegetation indices. The results show that the angle index composed by $525 \mathrm{~nm}, 555 \mathrm{~nm}$ and 670 $\mathrm{nm}$ can resist the effects of water columns and is more sensitive to vegetation parameters such as LAI. Furthermore, it makes a well discrimination between submerged vegetation and water bodies in the satellite data. We hope that the new index will provide a theoretical basis for future research.
\end{abstract}

\section{INTRODUCTION}

Submerged vegetation is an important part of wetland ecosystems, which refers to plants that live entirely below the water surface. These plants are ecologically essential because of their functions of reducing turbidity, absorbing heavy metal ions in water and inhibiting eutrophication of the water body. Therefore, it is of great significance to monitor the distribution and to assess the growth status of submerged vegetation for the protection of wetlands (Carpenter and Lodge, 1986). However, due to the underwater living environment, the conventional field surveys are inefficient with considerable expense. The technology of remote sensing allows rapid observation of a broad spatial range of wetlands, which is expected to provide great help to the monitoring of wetland ecosystems.

Currently, remote sensing has been widely utilized to retrieve the specific biophysical or biochemical parameters of terrestrial vegetation such as Leaf Area Index (LAI) (Gupta et al., 2000), biomass (Maire et al., 2011) and Fraction of Absorbed Photosynthetically Active Radiation (FPAR) (DailiangPeng et al., 2012). Many parameter inversion algorithms are constructed based on the vegetation index, which is a frequently-used tool for detecting vegetation. Vegetation index refers to the band combinations of satellite data whose purpose is to enhance the signal of vegetation while weakening the impact resulting from the background (Huete et al., 1994). At present, the indices such as Normalized Difference Vegetation Index (NDVI) (Rouse, 1974), Soil Adjusted Vegetation Index (SAVI) (Huete, 1988) and Enhanced Vegetation Index (EVI) (Huete et al., 1997) have been wildly used. However, the optical properties of water columns attenuate the vegetation signal seriously, which results in the totally different spectral characteristics of submerged vegetation. Those indices designed for the terrestrial vegetation are not suitable for the detection of submerged vegetation.
There has been some research relating to the construction of submerged vegetation index. Referenced to the concept of soilline, Villa etc. made use of the blue band and near infrared band of Landsat TM to construct the Normalized Difference Aquatic Vegetation Index (NDAVI) and Water Adjusted Vegetation Index (WAVI). After comparing the performance of the aquatic vegetation indices with those conventional ones, they found that the new vegetation indices have better capacity in distinguishing aquatic and terrestrial vegetation (Villa et al., 2014). The simulated data set used for assessment is obtained by a simple aquatic vegetation canopy model, which only considers the vegetation parameters such as LAI, leaf reflectance and leaf inclination distribution. A complex model, which also involves water parameters such as water depth and concentrations of water constituents, should provide more precise information, because those parameters are obviously influential in the spectral response of submerged vegetation. Zhou etc. proposed the Aquatic Vegetation Radiative Transfer model (AVRT) which takes vegetation, water parameters and observation conditions into account. The accuracy of AVRT has been evaluated by the in-situ spectral data and Monte Carlo simulation (Zhou et al., 2015). Hence, on the basis of AVRT, this paper tries to construct the submerged vegetation index, and assess whether the new index could meet the following requirements: (1) it should suppress the affection of water columns, (2) it should be sensitive to vegetation parameters and (3) it should provide good performance in distinguishing submerged vegetation and water bodies.

The rest of the paper is organized as follows: in Section 2, some characteristic bands chosen to build the submerged vegetation index are introduced, and the formulas of index and methods corresponding to the requirements are proposed as well. In Section 3, the simulated data and the satellite data used for

\footnotetext{
* Corresponding author
} 
analysis are presented. The results are stated in Section 4 and the conclusion is summarized in Section 5.

\section{METHODOLOGY}

\subsection{Band Selection}

According to the typical spectra of submerged vegetation (Figure 1 (a)) and its first order derivative (Figure 1 (b)), some characteristic bands are selected to build the new vegetation index:

(1) The maxima of spectra: $555 \mathrm{~nm}, 720 \mathrm{~nm}$ and $805 \mathrm{~nm}$, which represent the three peaks of the spectra;

(2) The minima of spectra: $439 \mathrm{~nm}, 670 \mathrm{~nm}$ and $740 \mathrm{~nm}$, which represent the three troughs of the spectra;

(3) The maxima of the first order derivative: $525 \mathrm{~nm}, 703 \mathrm{~nm}$ and $788 \mathrm{~nm}, 703 \mathrm{~nm}$ can be considered as the location of the "red edge" of submerged vegetation.

Hence there are nine characteristic bands altogether. The formulas of the new index are introduced next.
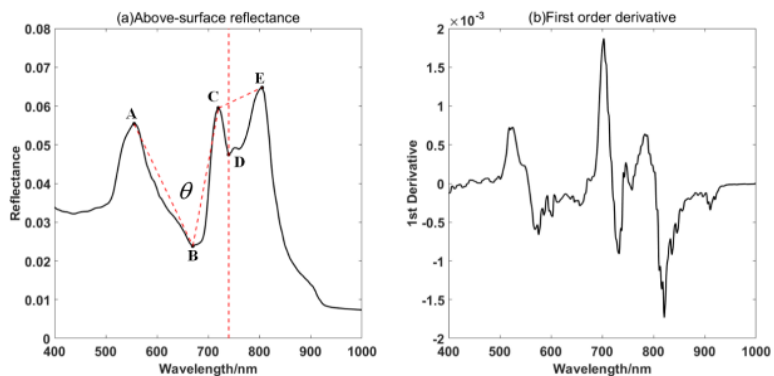

Figure 1. The typical above-surface reflectance of submerged vegetation (a) and the first order derivative spectra (b)

\subsection{The Formulas of Index}

2.2.1 The Slope Index: Taking two points A and B in Figure 1 (a) as an example, whose coordinates are supposed as $\left(\lambda_{A}, \rho_{A}\right)$ and $\left(\lambda_{B}, \rho_{B}\right)$, and the formulas of slope index is given by Equation (1). The slope index can represent the rate of increase or decrease between two characteristic bands. Nine bands can be combined to obtain thirty-six different slope indices.

$$
S L O P E=\frac{\rho_{B}-\rho_{B}}{\lambda_{B}-\lambda_{A}}
$$

2.2.2 The Absorption Index: The absorption characteristics can be described by the trough and two nearby peaks (Wang et al., 1996). Taking C, D and E in Figure 1 (a) as an example, where $\mathrm{C}$ and $\mathrm{E}$ locate in the peaks and $\mathrm{E}$ is in the trough, and the coordinates are $\left(\lambda_{C}, \rho_{C}\right) 、\left(\lambda_{D}, \rho_{D}\right)$ and $\left(\lambda_{E}, \rho_{E}\right)$, respectively. The line connecting $\mathrm{C}$ and $\mathrm{E}$ has a point $\left(\lambda_{D}, \rho_{R}\right)$, whose horizontal coordinate is the same as $\mathrm{D}$, and the absorption index is:

$$
\text { Absor }=\frac{\rho_{R}-\rho_{D}}{\rho_{R}}
$$

This index can be an indicator of the depth of absorption. According to Figure 1 (a), there are two pairs of combination that can be used to obtain the absorption index: 555-670-720 and $720-740-805$.

2.2.3 The Angle Index: The angle index refers to the angle between two lines where three points on the curve cross each other. Taking A, B and C in Figure 1 (a) as an example, the angle $\theta$ between line $\mathrm{AB}$ and $\mathrm{BC}$, which is calculated by the law of cosines, is used to obtain the angle index. To ensure the values are in the range of $0-1$, the angle should be divided by $\pi$. Besides, since the wavelengths (horizontal axis) are far greater than reflectance (vertical axis), the reflectance should be multiplied by a factor of 10000 . There are eighty-four kinds of angle index combined by the characteristic bands.

In this paper, the conventional vegetation indices NDVI and SAVI, and the aquatic vegetation indices NDAVI and WAVI are chosen as the comparison to assess the performance of the new indices. The formulas of these four indices are shown in Table 1 . The BLUE, RED and NIR bands in Table 1 correspond to $439 \mathrm{~nm}, 670 \mathrm{~nm}$ and $705 \mathrm{~nm}$, respectively.

\begin{tabular}{|l|c|}
\hline VI & Formula \\
\hline NDVI & $\frac{\rho(R E D)-\rho(N I R)}{\rho(R E D)+\rho(N I R)}$ \\
SAVI & $(1+L) \frac{\rho(R E D)-\rho(N I R)}{\rho(R E D)+\rho(N I R)+L}$ \\
NDAVI & $\frac{\rho(B L U E)-\rho(N I R)}{\rho(B L U E)+\rho(N I R)}$ \\
WAVI & $(1+L) \frac{\rho(B L U E)-\rho(N I R)}{\rho(B L U E)+\rho(N I R)+L}$ \\
\hline
\end{tabular}

Table 1. The formulas of NDVI, SAVI, NDAVI and WAVI

\subsection{Methods}

For the requirements mentioned in Section 1, these methods are selected to evaluate the performance of new indices:

(1) On the basis of AVRT, the simulation data, which describes the response of the submerged vegetation with various vegetation coverage (represented by LAI) living in different aquatic environment (represented by water depth, $\mathrm{Hw}$, and the concentration of chlorophyll a, Cchla), can be obtained. The standard deviation of new indices can be utilized to assess the capacity against the effects of water columns, because the standard deviation can reflect the degree of dispersion of the simulated data set. The smaller the standard deviation, the less discrete the data, and it indicates that the index is more powerful in resisting the effect of water columns.

(2) The Extended Fourier Amplitude Sensitivity Test (EFAST) method is utilized to analyze the global sensitivity of new indices to LAI, $\mathrm{Hw}$ and Cchla. If the new index is more sensitive to LAI and less sensitive to Hw and Cchla, this index is expected to achieve the requirements better. The global sensitivity analysis can elucidate the effect of each input parameters on the model output with a fully exploration of the input parameter space. The EFAST method proposed by Saltelli et al. can obtain the total effect of input parameters, which includes the effects of a single parameter and the interaction among parameters. This method achieves both accuracy and efficiency, and it can provide great help to this part of analysis (Saltelli et al., 1999). 
(3) An optical satellite image, which contains submerged vegetation and water bodies, is chosen to assess the performance of new index in distinguishing submerged vegetation and water. The region of interest (ROI) corresponding to the two kinds of objects are selected in the image, and the frequency histogram of new index in this two ROIs are plotted. According to the degree of coincidence of distribution ranges, the ability to discriminate between the two objects can be assessed. The lower the degree of coincidence, the better the discrimination ability of the index.

\section{DATA}

\subsection{Simulation Data}

There are various kinds of input parameters in AVRT. In this study, LAI is chosen to represent the vegetation coverage, and Hw and Cchla are chosen to simulate the variation of the water body. The reason for selecting these parameters as independent variables of the simulation data are that they are more representative than the others. The values of these parameters are shown in Table 2. The other parameters are set in reasonable default values. The sun zenith angle equals $30^{\circ}$, and the view zenith angle and the relative azimuth are taken as $0^{\circ}$. Each LAI corresponds to 64 kinds of aquatic environments, which are combined by Hw and Cchla, and a total of 2560 input samples can be obtained.

\begin{tabular}{|l|c|c|}
\hline Parameter & Value & Unit \\
\hline LAI & $0.1-4.0$, step $=0.1$ & {$[-]$} \\
Hw & $0.3-1.0$, step $=0.1$ & $\mathrm{~m}$ \\
Cchla & $0.1,1,5$, and $10-50$, step $=10$ & $\mathrm{mg} / \mathrm{m}^{3}$ \\
\hline
\end{tabular}

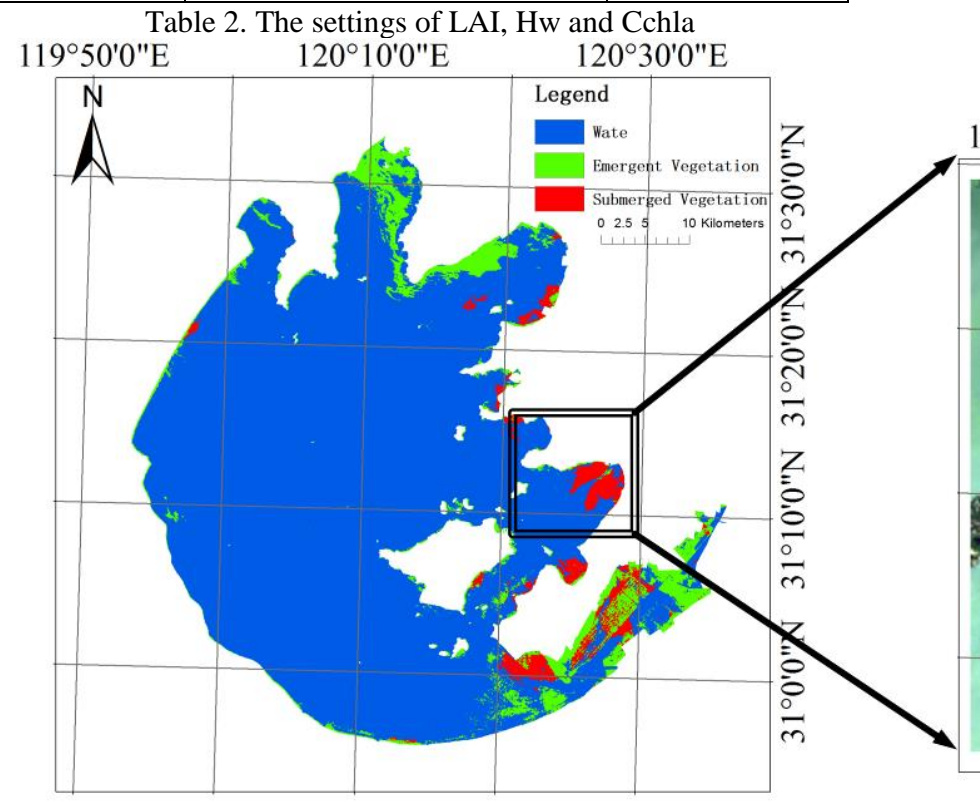

(a)

\subsection{Satellite Data}

According to Section 2.3, a scene of Sentinel-2A image in Taihu Lake, China, is chosen, which is acquired on April 29, 2017. Located in the southern part of the Yangtze River Delta, Taihu Lake is currently the third largest freshwater lake in China. The ecosystem of Taihu wetland has rich biodiversity. However, due to the influence of economic activities, the communities of aquatic vegetation in the lake have shown signs of obvious decline, and the stability of the ecosystem has been threatened. The supervision and protection of the Taihu Lake need immediate action. The Sentinel-2A satellite was launched on June 23, 2015. The satellite-mounted multispectral imager has multiple bands in the near-infrared region, which covers some characteristic bands of aquatic vegetation. The imager has high spatial resolution and is suitable for detecting wetland aquatic vegetation whose living environments are complex. Therefore, the remote sensing data of Sentinel-2A has great potential for application in wetland monitoring.

Due to the lack of field-based surveys, a classification data set of aquatic vegetation in Taihu Lake, which is generated from 2011 to 2013, are chosen as the reference (Ma et al., 2015). The region classified as submerged vegetation and the region classified as water bodies are selected in the eastern part of Taihu Lake. The classification data and the processed Sentinel2A image are shown in Figure 2, they are generated in the same season, and the spatial resolution in (a) and (b) are $30 \mathrm{~m}$ and 10 $\mathrm{m}$, respectively. In Figure 2 (b), the red and blue region are classified as submerged vegetation and water bodies, and there are 24639 and 24585 pixels in these two ROIs, respectively.

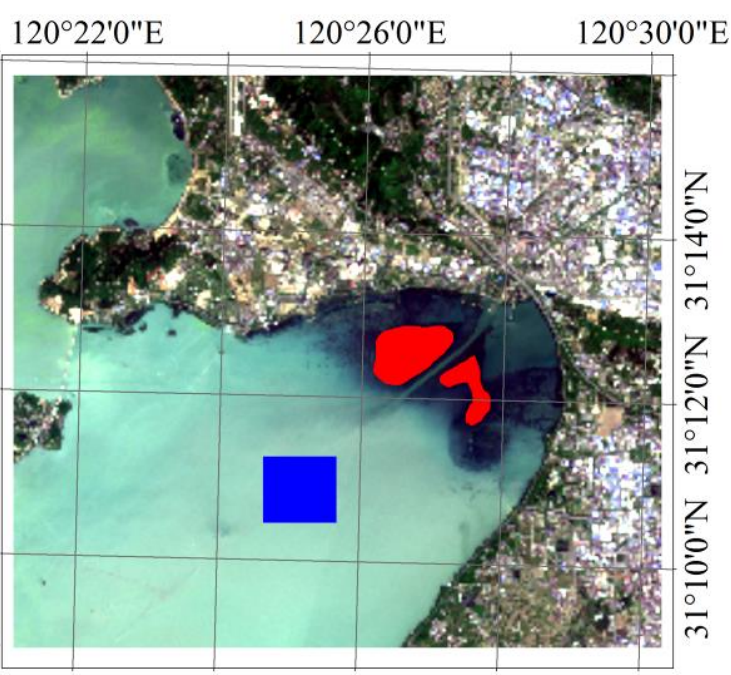

(b)

Figure 2. The remote sensing data used in the analysis. (a) The classification data set of aquatic vegetation in Taihu Lake, where the blue, green and red regions are classified as water, emergent vegetation and submerged vegetation, respectively. (b) The eastern part of Taihu Lake in the satellite image of Sentinel-2A, where the red and blue regions are classified as submerged vegetation and water bodies, respectively 


\section{RESULTS}

\subsection{The Standard Deviation}

For every vegetation index involved, the total standard deviations of the 2560 samples are calculated, which are sorted in an ascending order. For the slope indices and the angle indices mentioned in this paper, the top five indices and their standard deviations are shown in Table 3 . All of the absorption indices, and the indices used for comparison in Table 2, are also included in Table 3.

\begin{tabular}{|l|c|c|c|c|c|}
\hline Slope Index & $439-670$ & $525-670$ & $439-525$ & $439-555$ & $555-670$ \\
\hline Total STD & 0.016 & 0.156 & 0.251 & 0.381 & 0.392 \\
\hline Angle Index & $439-525-555$ & $740-788-805$ & $439-525-670$ & $439-670-703$ & $525-555-670$ \\
\hline Total STD & 0.009 & 0.046 & 0.055 & 0.056 & 0.059 \\
\hline Absorption Index & \multicolumn{3}{|c|}{$555-670-720$} & \multicolumn{3}{c|}{0.173} \\
\hline Total STD & \multicolumn{2}{|c|}{$0.173-740-805$} \\
\hline Index for comparison & NDVI & SAVI & \multicolumn{2}{c|}{ NDAVI } & WAVI \\
\hline Total STD & 0.116 & 0.028 & \multicolumn{2}{c|}{0.123} & 0.028 \\
\hline
\end{tabular}

Table 3. The total standard deviations of different indices

As can be seen from Table 3, the total standard deviations of the angle indices are all small, whereas the standard deviations of the two absorption indices are much larger than all of the others Among the slope indices, the combination of $439-670$ is the only one whose standard deviation is lower than 0.1. The standard deviations of the normalized indices (NDVI and NDAVI) are larger than 0.1 , whereas they are about 0.02 for the adjusted indices (SAVI and WAVI). For each index mentioned in Table 3, the patterns of total standard deviations variation as function of LAI are plotted in Figure 3.

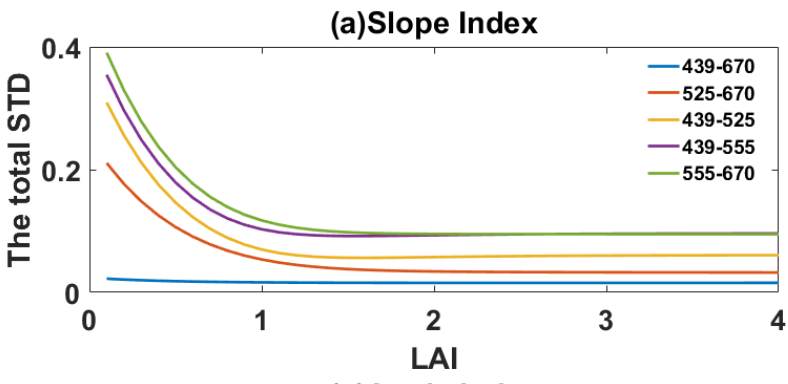

(c)Angle Index

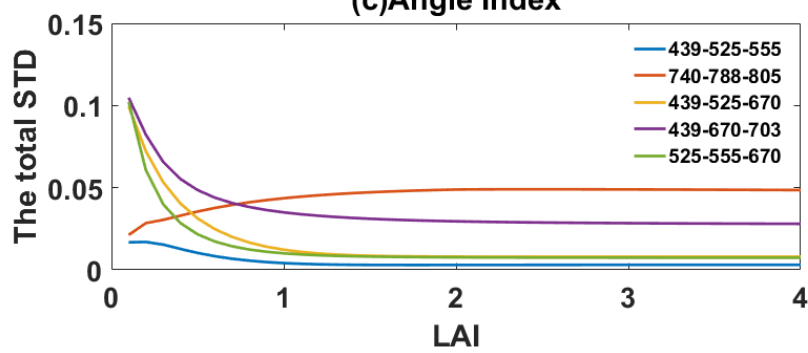

It can be seen from Figure 3 that for all of the absorption indices and the indices used for comparison, the total standard deviations are increased with LAI. The angle index 740-788805 has the same pattern as well. It indicates that the ability of these indices to resist the effects of water columns are weakened as vegetation coverage increases, but the results expected is obviously the opposite. As LAI increases, it is expected to receive more signals of vegetation and fewer signals resulting from water columns, which indicates that the observed response of submerged vegetation should be gradually stable.

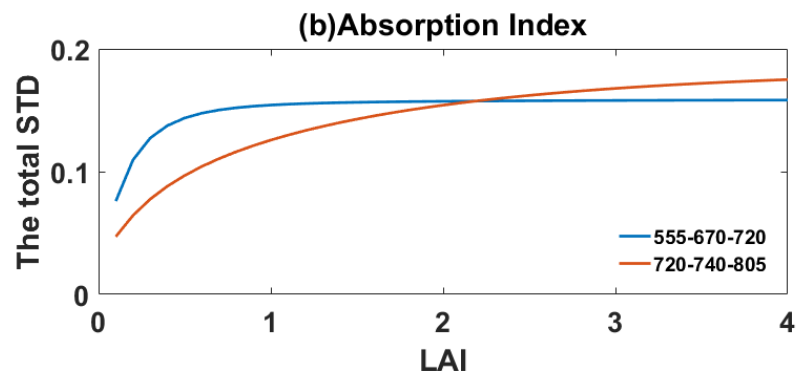

(d)Index for comparison

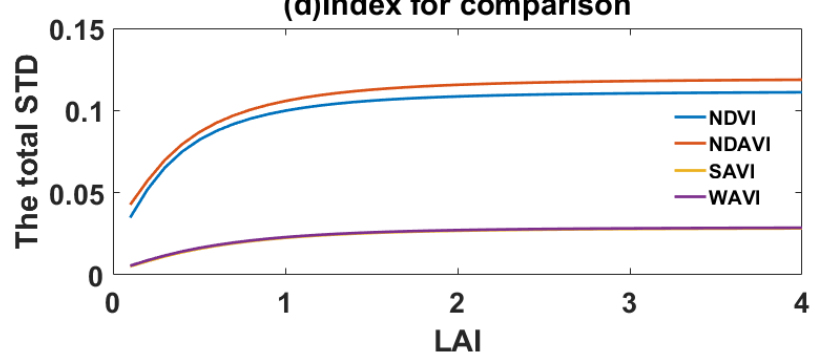

Figure 3. The patterns of total standard deviations variation as function of LAI for different indices. (a): Slope index; (b) Absorption index; (c) Angle index; (c) Index for comparison

In summary, the slope index 439-670, and all of the angle indices are selected for the next step of analysis. To ensure the analysis is complete, the absorption index 720-740-805 is also included as it has lower standard deviation than the other absorption index.

\subsection{Global Sensitivity Analysis}

For the indices selected in Section 3.1, the global sensitivity analysis is performed. The input samples needed by EFAST method are generated on the basis of the distributions and ranges of input parameters. The ranges of LAI, Hw and Cchla are the same as those in Table 2, and the distributions are assumed as uniform as there are no prior knowledge about them. According to the sampling strategy of EFAST, a total of $513 \times 3=1539$ samples are generated, where 513 means the number of samples needed for a single input parameter, and 3 is the number of parameters in the analysis. The number of samples is determined by comparison of the results of different sample size, and the better one should be computationally efficient and robust. The results of EFAST are shown in Table 4. The values in the table are the total sensitivity index, which are 
the measurement of the effects imposed to the output by the input parameters.

\begin{tabular}{|l|c|c|c|}
\hline Vegetation Index & $\mathrm{Hw}$ & Cchla & LAI \\
\hline Slope 439-670 & 0.689 & 0.742 & 0.114 \\
Angle 439-525-555 & 0.818 & 0.406 & 0.725 \\
Angle 740-788-805 & 0.979 & 0.019 & 0.128 \\
Angle 439-525-670 & 0.174 & 0.213 & 0.941 \\
Angle 439-670-703 & 0.607 & 0.186 & 0.518 \\
Angle 525-555-670 & 0.162 & 0.266 & 0.954 \\
Absorption 555-670-720 & 0.953 & 0.015 & 0.130 \\
NDVI & 0.791 & 0.028 & 0.252 \\
SAVI & 0.807 & 0.019 & 0.250 \\
NDAVI & 0.796 & 0.029 & 0.246 \\
WAVI & 0.811 & 0.019 & 0.244 \\
\hline
\end{tabular}

Table 4 . The results of global sensitivity analysis

The new index is expected to be more sensitive to LAI, which means it is more possible to retrieve LAI by this index, and to be less sensitive to $\mathrm{Hw}$ and Cchla. Therefore, it can be seen from Table 4 that the angle indices 439-525-670 and 525-555670 perform better than the others. The sensitivities of normalized indices and adjusted indices are derived as well, whereas their sensitivities to LAI are much lower than that to the water parameters.

According to the results in Section 3.1 and 3.2, compared with the other indices involved, the angle indices 439-525-670 and 525-555-670 have lower standard deviations, and they are more sensitive to LAI. It can be summarized that these indices are more suitable than the conventional terrestrial vegetation

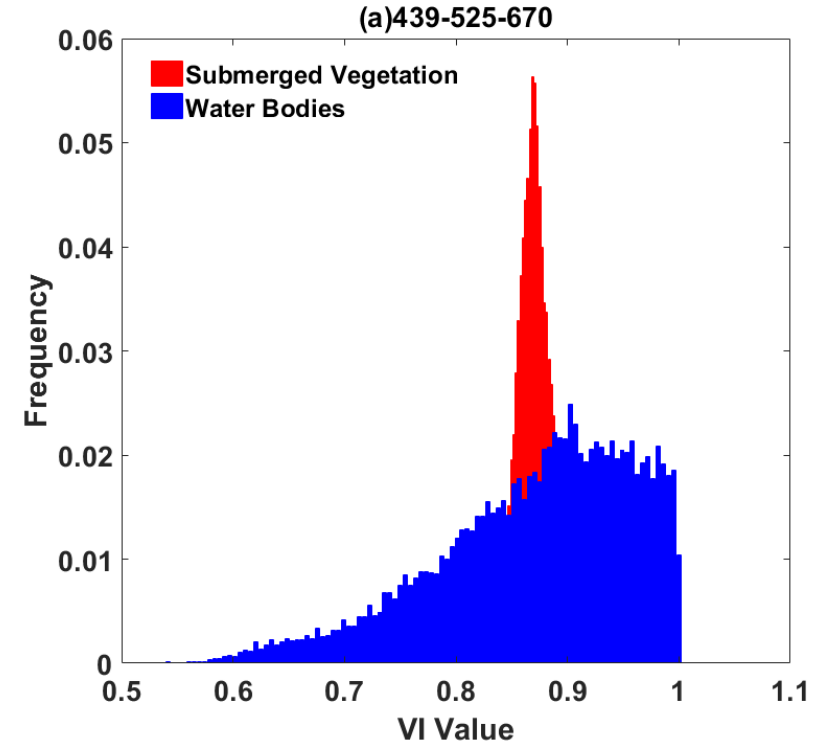

indices (NDVI and SAVI) and the aquatic vegetation indices (NDAVI and WAVI) in the applications of wetland remote sensing. Therefore, the capacities of these two angle indices in distinguishing submerged vegetation and water are assessed in Section 3.3 .

\subsection{The Comparison by Satellite Data}

The image used in this study is pre-processed, which includes radiometric correction and atmospheric correction. The band centre wavelengths are $443 \mathrm{~nm}, 490 \mathrm{~nm}, \quad 560 \mathrm{~nm}, 665$ nm, $705 \mathrm{~nm}, 740 \mathrm{~nm}, 783 \mathrm{~nm}, \quad 865 \mathrm{~nm}, 945 \mathrm{~nm}, 1610$ $\mathrm{nm}$ and $2190 \mathrm{~nm}$, respectively. For the sake of simplicity, they are represented by B1-B11. According to the spectral response functions of Sentinel-2A, the characteristic bands in $439 \mathrm{~nm}$, $525 \mathrm{~nm}, 555 \mathrm{~nm}$ and $670 \mathrm{~nm}$ corresponds to B1, B2, B3 and B4, respectively, in the following analysis.

The two ROIs specified to submerge vegetation and water are shown in Figure 2 (b), and the angle indices 439-525-670 and 525-555-670 are calculated. The frequency histogram of the indices' values are plotted in Figure 4. For submerged vegetation, the values of angle index 525-555-670 are mainly in the range of 0.55-0.85, whereas the values are in the range of $0.17-0.25$ for water bodies. As for angle index 439-525-670, the values for submerged vegetation and water bodies are in the range of 0.84-0.92 and 0.74-0.98, respectively, which means the ranges are partly overlapped. Obviously, the angle index 525555-670 performs better than the other since its distribution ranges for submerged vegetation and water bodies are totally different.

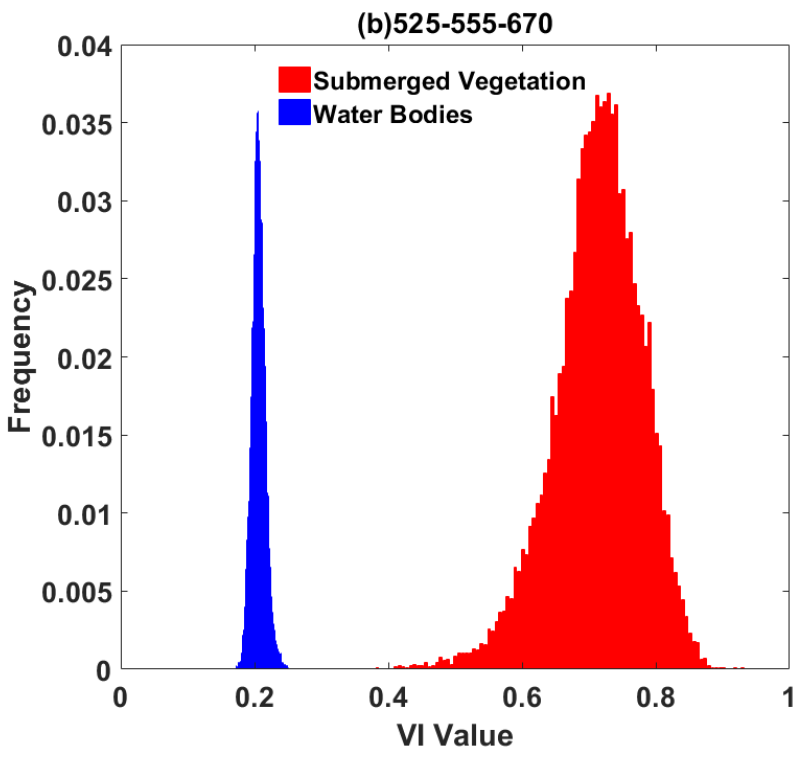

Figure 4. The frequency histograms of the angle indices for submerged vegetation (red) and water bodies (blue). (a) 439-525-670; (b) 525-555-670

\section{CONCLUSION}

On the basis of AVRT model, some characteristic bands were selected based on the spectral properties of submerged vegetation. The new submerged vegetation index was constructed in the form of slope index, angle index and absorption index. The standard deviations of these indices were calculated from the simulation data, and the sensitivities of the indices to LAI, the height of the water body and the concentration of chlorophyll a were analyzed. Determined from the results, the angle indices composed by $439 \mathrm{~nm}, 525 \mathrm{~nm}$, and $670 \mathrm{~nm}$, and by $525 \mathrm{~nm}, 555 \mathrm{~nm}$ and $670 \mathrm{~nm}$ perform better than other indices. They have lower standard deviations and are more sensitive to LAI. Based on a scene of Sentinel-2A image in Taihu Lake, China, these two indices' abilities to discriminate between submerged vegetation and water bodies were assessed. The regions classified as submerged vegetation and water bodies were selected form the image, and the frequency histograms of the two indices in the regions selected were plotted. The results show that the angle index 525-555- 
670 makes a better distinction between submerged vegetation and water bodies. We hope that the new submerged vegetation index can provide inspirations for the research of other scholars and provide a theoretical basis for the construction of wetland parameter inversion algorithms.

\section{ACKNOWLEDGEMENTS}

This work was supported by the following funds: Beijing Natural Science Foundation (Grant No. 8162028); National Natural Science Foundation of China (Grant No.40901168); CETC key laboratory of aerospace information applications (Grant No. XX17629X009); National Key Research and Development Program of China (Grant No. 2016YFC0201800); Beijing Municipal science \& technology commission (Grant No. Z171100000717010). Acknowledgement for the data support from "National Earth System Science Data Sharing Infrastructure, National Science \& Technology Infrastructure of China. (http://www.geodata.cn)".

\section{REFERENCES}

Carpenter, S.R. and Lodge, D.M., 1986. Effects of submersed macrophytes on ecosystem processes. Aquatic Botany, 26(3-4): 341-370.

DailiangPeng, BingZhang and LiangyunLiu, 2012. Comparing spatiotemporal patterns in Eurasian FPAR derived from two NDVI-based methods. International Journal of Digital Earth, 5(4): 283-298.

Gupta, R.K., Prasad, T.S. and Vijayan, D., 2000. Relationship between LAI and NDVI for IRS LISS and Landsat TM bands. Advances in Space Research, 26(7): 1047-1050.

Huete, A.R., 1988. A soil-adjusted vegetation index (SAVI). Remote Sensing of Environment, 25(3): 295-309.

Huete, A., Justice, C. and Liu, H., 1994. Development of vegetation and soil indices for MODIS-EOS. Remote Sensing of Environment, 49(3): 224-234.
Huete, A.R., Liu, H.Q., Batchily, K. and Leeuwen, W.V., 1997. A comparison of vegetation indices over a global set of TM images for EOS-MODIS. Remote Sensing of Environment, 59(3): 440-451.

Ma, R. et al., 2015. Spatial Distribution Data of Aquatic Vegetation in Taihu Lake, 2011-2013. National Earth System Science Data Sharing Infrastructure, National Science \& Technology Infrastructure of China http://www.geodata.cn.

Maire, G.L. et al., 2011. MODIS NDVI time-series allow the monitoring of Eucalyptus plantation biomass. Remote Sensing of Environment, 115(10): 2613-2625.

Rouse, J.W., 1974. Monitoring the vernal advancement and retrogradation (greenwave effect) of natural vegetation. Nasa, USA.

Saltelli, A., Tarantola, S. and Chan, P.S., 1999. A Quantitative Model-Independent Method for Global Sensitivity Analysis of Model Output. Technometrics, 41(1): 39-56.

Villa, P., Mousivand, A. and Bresciani, M., 2014. Aquatic vegetation indices assessment through radiative transfer modeling and linear mixture simulation. International Journal of Applied Earth Observation \& Geoinformation, 30(1): 113127.

Wang, J.N., Zheng, L.F. and Tong, Q.X., 1996. The spectral absorption identification model and mineral mapping by imaging spectrometer data. Remote Sensing of Environment, 11(1): 20-31.

Zhou, G. et al., 2015. Canopy modelling of aquatic vegetation: A radiative transfer approach. Remote Sensing of Environment, 163: 186-205. 